\title{
Bekenntnis zur Nachhaltigkeit
}

\author{
Nachhaltige Enfwicklung ist seit der Konferenz in Rio 1992 zum politischen \\ Leitbegriff geworden. Auch wenn dies größtenteils unbemerkt von einer breite- \\ ren Öffentlichkeit passiert, so gewinnt das Thema gerade international deutlich \\ an Fahrt. Um ihrer zunehmenden gesellschaftlichen Verantwortung gerecht zu \\ werden, haben sich Unternehmen aus allen Teilen der deutschen Wirtschaft \\ kürzlich im Forum Nachhaltige Entwicklung zusammengeschlossen.
}

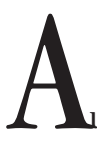

A Von Wolf-Eberhard Schiegl und Ralph Thurm des Umweltschutzes in andere Politikbereiche seit dem Europäischen Rat in Cardiff ein Kernthema auf der politischen Agenda. International wird die Rio+10-Konferenz im Jahr 2002 in Südafrika ein Markstein für die Weiterentwicklung des Nachhaltigkeitsleitbildes - hoffentlich mit wegweisenden Impulsen für die Zukunft. In Deutschland zeigt die Planung der Bundesregierung für eine nationale Nachhaltigkeitsstrategie bisher hingegen noch kaum Wirkung.

In vielen in Deutschland beheimateten und international aktiven Unternehmen findet die Integration der drei Dimensionen der nachhaltigen Entwicklung, Ökonomie, Ökologie und Soziales, bereits sichtbar statt; sie ist vielfach Teil des Firmenleitbildes und damit Aufgabe für die Unternehmenspolitik geworden. Dies bedeutet, neben den wirtschaftlichen Aspekten auch das ökologische Profil und die gesellschaftlichen Auswirkungen von Produkten und Produktionsprozessen zu einem wichtigen Entscheidungskriterium zu machen; und dies entlang der gesamten Wertschöpfungskette in Unternehmen der Industrie, bei Dienstleistern und Handel, aber auch bei Entscheidungen von Banken und Versicherungen.

Die Integration muss nun aber endlich auch in der Politik manifest werden, da viele Aufgaben mit den etablierten Konzepten einzelner Politikfelder nicht mehr zu lösen sind: Klimapolitik ist nicht nur Umweltpolitik, die isoliert mit Auflagen, Grenzwerten und Steuern betrieben werden kann, sondern ebenso Wirtschafts- und Sozialpolitik sowie auch Verkehrspolitik. Das Austarieren der zum Teil gegenläufigen Effekte verschiedenster Politikbereiche macht eine übergeordnete Strategie auch für die Politik unabdingbar. Vor dem Hintergrund dieser bisher ungelösten Aufgabe der Politik zeigt sich den Unternehmen, die sich als ,Corporate Citizen“ verstehen, deutlich: Eine Steigerung der Energieeffizienz der Produktion wird ebenso wie die Optimierung des Produktlebenszyklus oder nachhaltige Mobilität nicht auf dem Verordnungswege erreicht, sondern durch Innovation in Technik und Management.

\section{- Neve Verantwortung der Unternehmen}

Die Lösungskompetenz der Unternehmen muss deshalb nicht wie bisher erst bei der technischen Umsetzung staatlich gesetzter Vorgaben zum Zuge kommen, sondern unmittelbar im Entwicklungsprozess neuer politischer Instrumente. Es muss das Ziel sein, realistische und somit unternehmerisch tragfähige Ziele zu definieren, diese mit den geeigneten Instrumenten eigenverantwortlich umzusetzen und anschließend den glaubwürdigen Nachweis der Zielerreichung gegenüber der Öffentlichkeit anzutreten.

Die Tatsache, dass sich viele Unternehmen dieser neuen Rolle in der Gesellschaft deutlich bewusst sind, soll mit der Gründung des ,Forum Nachhaltigkeit der deutschen Wirtschaft" zum öffentlich sichtbaren Bekenntnis werden. Diese neue Institution soll die politische Lücke zwischen neuen Aufgaben und alten Konzepten durch von der Wirtschaft entwickelte, praktisch umsetzbare, sich betriebswirtschaftlich rechnende und effiziente Vorschläge schließen. Das Forum Nachhaltigkeit soll als Denkfabrik der Wirtschaft zu Fragen der Nachhaltigkeit den institutionellen Rahmen schaffen, in dem neue Ideen und Konzepte von der Wirtschaft entwickelt und in die Diskussion mit der Politik eingebracht werden. Frühe Kommunikation und Unterstïtzung gemäß der Maxime ,we have to be in it to win it" mit der Politik anstelle bloßer Reaktion auf fertige Ideen ist der zentrale Erfolgsfaktor.

In diesem Forum wird die ganze Kompetenz der mitwirkenden Unternehmen zur Entwicklung wirksamer Vorschläge für die Politik gebündelt und genutzt. Zugleich soll das Forum die zentrale Plattform für die Diskussion zwischen der Wirtschaft und den politischen Entscheidungsträgern über ein zukunftsfähiges Wirtschaften werden. Über die nationale Ebene hinaus kann damit auch die Position der deutschen Wirtschaft in der internationalen Diskussion gestärkt werden, ob bei den Vereinten Nationen, der Europäischen Union oder der OECD, nicht zuletzt aber auch im Dialog mit dem etablierten Vorreiter unternehmerischer Nachhaltigkeitskompetenz, dem World Business Council for Sustainable Development (WBCSD).

Zunehmend erweisen sich technologische Lösungskompetenz, aber auch die Fähigkeit zur Organisation von Veränderungsprozessen, als Engpassfaktoren der Nachhaltigkeit. Hier sind die Unternehmen in Zukunft mehr denn je gefordert. Dem Leistungsnachweis in Sachen Nachhaltigkeit kommt deshalb eine Schlüsselbedeutung zu.

\section{Das Forum: Arbeitsweise ...}

Das Forum Nachhaltige Entwicklung ist ein Zusammenschluss von Unternehmen aus allen Teilen der Wirtschaft. Ziel ist es, über die Gründungsmitglieder hinaus, ca. 40 bis 50 Unternehmen für die Mitgliedschaft im Forum zu gewinnen. Diese legen mit der Mitgliedschaft ihrer Vorstandsvorsitzenden im Kuratorium des Forums ein klares Bekenntnis in Sachen nachhaltige Entwicklung ab.

Folgende Firmen und Verbände sind bereits Mitglied des Forums: Allianz, BASF, Bayer, BMW, Bosch, DaimlerChrysler, Gerling, Heidelberger Zement, Henkel, Preussag, Ruhrgas, Ruhrkohle, RWE, Eon, Siemens, Tetra Pak, der VCI sowie VW. Das Arbeitsmuster des Forums lässt sich mit einem Dreischritt beschreiben:

1. Denken: Als Denkfabrik der Wirtschaft will es Sachkompetenz bündeln und frühzeitig Trends der Nachhaltigkeit erkennen und analysieren.

2. Handeln: Als „Ingenieurbüro“ will es neue Ideen experimentell durchspielen und ihre Realisierungschancen erproben.

3. Kommunizieren: Als Dialogforum will es sowohl innerhalb der Wirtschaft als auch gegenüber der Politik und den gesellschaftlichen Gruppen Anforderungen an die künftigen Leistungen von Unternehmen identifizieren und diskutieren.

\section{... und Aufgaben}

Die Aufgaben, die das Forum Nachhaltigkeit dabei im Einzelnen angehen soll, lauten:

- Trends der internationalen, der europäischen und der deutschen Nachhaltigkeitsdebatte frühzeitig erkennen und analysieren. 
Hochrangige Diskussionen mit politischen Entscheidungsträgern im Vorfeld konkreter politischer Vorschläge organisieren.

- Wirtschaftskompetenz gegenüber der (Fach-) Öffentlichkeit anhand von Beispielen aus der Praxis glaubhaft und überzeugend darstellen.

- Schnelle Entwicklung realistischer Vorschläge in thematisch fokussierten Projektteams.

- Präsentation von Vorschlägen gegenüber der Politik, speziell zu einzelnen Themenbereichen (,Themen besetzen“).

- Ermittlung der Möglichkeiten für eine einvernehmliche Lösung im Dialog - Früherkennung unabwendbarer Konflikte.

- Präsenz in nationalen und internationalen Gremien; Organisation von Side-Events, fokussiert auf exemplarische Leistungen von Unternehmen.
- Information in die Verbände der Wirtschaft über neue Themen und deren mögliche Lösung (,Initiatorfunktion“).

Im Vordergrund stehen ganz bewusst die Querschnittsthemen, die direkt die Arbeit in den Unternehmen betreffen und für die in den kommenden Jahren die „Spielregeln“ entwickelt werden. Beispiele gibt es hierfür genug: Von der Quantifizierung der Nachhaltigkeit - Wie können Leistungen der Unternehmen unter Nachhaltigkeitsgesichtspunkten gemessen werden? - über die Zukunft des Vorsorgeprinzips - Wie wird verantwortliches Handeln unter Unsicherheit nachgewiesen? - reicht das Spektrum bis zur Klimapolitik und den Werkzeugen für nachhaltige Produkte und nachhaltigen Konsum. Zur Umsetzung steht dabei das gesamte Spektrum an Instrumenten zur Verfügung.
Die Voraussetzungen der deutschen Wirtschaft als wichtiger Akteur in der Nachhaltigkeitsdiskussion, ob national oder international, sind gut. Die Arbeit hat allerdings erst richtig begonnen. Die Chancen sind besser denn je - nutzen wir sie!

\section{Die Autoren}

Dr. Wolf-Eberhard Schiegl ist Leiter der Unternehmensreferate Umweltschutz, Technische Sicherheit des SiemensKonzerns und Mitglied im Lenkungskreis des Forums Nachhaltige Entwicklung der deutschen Wirtschaft. Ralph Thurm ist Referent im Unternehmensreferat Betrieblicher Umweltschutz und Leiter eines internen Fachteams Nachhaltige Entwicklung.

Kontakt: Siemens AG, Corporate Offices Environmental Protection, Technical Safety, 81730 München. Tel. 089/636-42241, Fax -40162, E-mail: ralph. thurm@mchp.siemens.de

\section{Standard für soziale Verantwortung}

M Von Achim Lohrie it der internationalen Umweltkonferenz in Rio 1992 ist das Prinzip der Nachhaltigkeit global zu einem Leitbild für die zukünftige wirtschaftliche und gesellschaftliche Entwicklung geworden. Obwohl die Forderung nach Nachhaltigkeit in erster Linie immer an Hersteller und Verbraucher gerichtet wird, kann auch der Handel als Mittler zwischen Angebot und Nachfrage einen Beitrag zur Nachhaltigkeit leisten, nämlich:

- im Sinne der ökonomischen Nachhaltigkeit eine Entwicklung forcieren, die wirtschaftliche Prosperität und Vollbeschäftigung auch für kommende Generationen ermöglicht,

- im Sinne der ökologischen Nachhaltigkeit die Naturressourcen nur in dem Maße nutzen, dass ihr Potenzial auch zukünftigen Generationen zur Verfügung steht, und schließlich
Vorangegangen war die durch internationale Verbraucherkampagnen erfolgte Sensibilisierung der Öffentlichkeit über die Verletzung elementarer Arbeits- und Sozialrechte in den weltweiten Produktionsstätten von Konsumgütern.

Ziel der Initiative ist es, ein weltweit gültiges Zertifizierungs- und Kontrollsystem für Sozialstandards auf der Ebene der betroffenen Produktionsstätten zu etablieren. Analog zu den bekannten Zertifizierungsprozessen für Qualität und Umweltmanagement - den ISO-Normen 9000 und 14.000 - sollen Produktionsbetriebe so die Möglichkeit erhalten, sich die Einhaltung sozialer Mindeststandards von unabhängigen Institutionen bestätigen zu lassen. Die Einhaltung wird extern von Zertifizierungsunternehmen nach klaren, bekannten und nachvollziehbaren Regeln und Gesetzen kontrolliert.

Otto wurde bereits 1996 vom Council on Economic Priorities (nach Umfirmierung heute: Social Accountability International, SAI) aufgefordert, sein Know-how in den Entwicklungsprozess einzubringen und das System in Pilotanwendungen bei seinen Lieferanten auf Praktikabilität und Durchsetzbarkeit zu überprüfen.

\section{Die Elemente des SA 8000}

Das Basisdokument des SA 8000 ist der Sozialstandard. Er definiert die Anspruchsgrundlage für den Zertifizierungsprozess und legt die geforderten sozialen Mindestanforderungen fest. Im Einzelnen sind dies 
(c) 20I0 Authors; licensee IÖW and oekom verlag. This is an article distributed under the terms of the Creative Commons Attribution Non-Commercial No Derivates License (http://creativecommons.org/licenses/by-nc-nd/3.o/), which permits unrestricted use, distribution, and reproduction in any medium, provided the original work is properly cited. 